\title{
Stimulus-response complexity influences task-set inhibition in task switching
}

\author{
Li Zhao ${ }^{\text {Equal first author, } 1,2,3}$, Saisai Hu ${ }^{\text {Equal first author, 1, 2, } 3}$, Yingying Xia ${ }^{1,2,3}$, Jinyu Li ${ }^{1,2,3}$, Jingjing Zhao ${ }^{1,2,3}$, Ya Li ${ }^{1,2,3}$, Yonghui \\ Wang ${ }^{\text {Corresp. 1, 2, } 3}$ \\ ${ }^{1}$ School of Psychology, Shaanxi Normal University, Xi'an, China \\ 2 Shaanxi Provincial Key Laboratory of Behavior \& Cognitive Neuroscience, Xi'an, China \\ 3 Shaanxi Provincial Key Research Center of Child Mental and Behavioral Health, Xi'an, China \\ Corresponding Author: Yonghui Wang \\ Email address: wyonghui@snnu.edu.cn
}

Previous studies have found that inhibiting a task set plays an important role in task switching. However, the impact of stimulus-response(S-R) complexity on this inhibition processing has not been explored. In this study, we applied the backward inhibition paradigm (switching between tasks $A, B$, and $C$, presented in sets of three) in order to investigate inhibition performance under different $S-R$ complexities caused by corresponding S-R mappings. The results showed that the difficult condition resulted in a greater switch cost than the moderate and easy conditions. Furthermore, we found a significant $n-2$ repetition cost under the easy S-R complexity that was reversed under the difficult S-R complexity. To verify stability of the reversed $n-2$ repetition cost in the difficult condition, we recruited another independent sample to conduct an additional experiment with the difficult condition. We also replicate a reversed $n-2$ repetition cost. These findings suggest that S-R complexity affects task-set inhibition in task switching because the effect of the task-set inhibition was insignificant when the S-R complexity increased; it was only significant under the easy condition. This result was caused by the different cognitive resource assignments. 


\section{Stimulus-response complexity influences task-set inhibition in task switching}

4 Li Zhao ${ }^{1,2,3}$, Saisai $\mathrm{Hu}^{1,2,3}$, Yingying Xia ${ }^{1,2,3}$, Jinyu $\mathrm{Li}^{1,2,3}$,Jingjing Zhao ${ }^{1,2,3}, \mathrm{Ya} \mathrm{Li}^{1,2,3}$, Yonghui $5 \quad$ Wang $1,2,3$

$6 \quad{ }^{1}$ School of Psychology, Shannxi Normal University, China

$7 \quad{ }^{2}$ Shaanxi Provincial Key Laboratory of Behavior \& Cognitive Neuroscience, China

$8{ }^{3}$ Shaanxi Provincial Key Research Center of Child Mental and Behavioral Health, China

\section{Li Zhao and Saisai Hu contributed equally to this work.}

*Corresponding Author:

Yonghui Wang, School of Psychology, Shaanxi Normal University, Xi'an, China

E-mail: wyonghui@snnu.edu.cn

\section{Abstract}

Previous studies have found that inhibiting a task set plays an important role in task switching.

However, the impact of stimulus-response(S-R) complexity on this inhibition processing has not been explored. In this study, we applied the backward inhibition paradigm (switching between tasks A, B, and C, presented in sets of three) in order to investigate inhibition performance under different S-R complexities caused by corresponding S-R mappings. The results showed that the difficult condition resulted in a greater switch cost than the moderate and easy conditions. Furthermore, we found a significant $n-2$ repetition cost under the easy S-R complexity that was reversed under the difficult S-R complexity. To verify stability of the reversed n-2 repetition cost in the difficult condition, we recruited another independent sample to conduct an additional 
25 experiment with the difficult condition. We also replicate a reversed n-2 repetition cost. These

26 findings suggest that S-R complexity affects task-set inhibition in task switching because the

27 effect of the task-set inhibition was insignificant when the S-R complexity increased; it was only

28 significant under the easy condition. This result was caused by the different cognitive resource

29 assignments.

\section{Introduction}

31 People must be highly adaptable and flexible when faced with complex environmental changes.

32 This often involves engaging in complex cognitive control processes in order to more actively

33 perform tasks and achieve goals. Task switching is a concept that has been well-established in

34 prior studies on cognitive control (Monsell, 2003; Kiesel et al., 2010; Vandierendonck,

35 Liefooghe, \& Verbruggen, 2010). Classic task-switching studies usually involve at least two

36 tasks with specific explanations regarding what task to do at what time and which procedures or

37 rules apply to the stimulus. A switch sequence is defined as two consecutive trials for different

38 tasks, and a repeat sequence is defined as two consecutive trials for the same task. Previous

39 studies have found that people respond more slowly and less accurately to switch sequences than

40 repeat sequences, reflecting the switch cost (Rogers \& Monsell, 1995; Monsell, 2003; Meiran,

41 2010). Rogers and Monsell's (1995) study included two tasks where either the numerical

42 member of a pair of characters was classified as even/odd, or the letter member was classified as

43 a consonant/vowel. Their results showed a large asymptotic reaction time (RT) cost. Typically, a

44 task set, or a specific configuration of the cognitive system, is needed to perform a task.

45 In a typical task-cueing paradigm, a trial consists of a task cue, a stimulus, and a response. The

46 task cue activates the task set, the task set is applied to the stimulus to gain a response, and the

47 response is typically activated via an arbitrary (and recently learned) stimulus (i.e., response 
48 mapping) (Grange \& Houghton, 2010). A task set is defined as a collection of control settings or

49 task parameters that program a system to perform processes such as stimulus identification,

50 response selection, or response execution (Vandierendonck, Liefooghe, \& Verbruggen, 2010).

51 Task sets are also assumed to include a representation of a task goal, a set of task-relevant stimuli,

52 a set of possible responses, and a mapping of the stimulus or stimulus categories to the responses

53 (Koch et al., 2010). Therefore, a task set includes two aspects: a stimulus set and a response set.

54 The stimulus set represents stimulus-related processes such as stimulus encoding and

55 identification. The response set represents response-related processes such as relevant stimulus-

56 response (S-R) mapping and activation of the correct response category (e.g., left or right) or

57 modality (e.g., finger or foot)(Rogers \& Monsell, 1995; Schuch \& Koch, 2004; Philipp \& Koch,

58 2005). For example, in a color judgment task, a left key press may mean "red", but in a size

59 judgment task, a left key press may mean "small”. Therefore, S-R mapping constitutes the

60 cognitive meaning of a response, which is the link between a stimulus and a response (Gade \&

61 Koch, 2007). Changing the correspondence of the S-R mapping and the value of the stimulus

62 would affect the S-R complexity. For example, univalent mappings link one dimension of a

63 stimulus with a response key, while bivalent mappings link two dimensions of a stimulus with a

64 response key.

65 Several previous studies considered the effect of inhibiting the task set during task switching by

66 applying the backward inhibition paradigm (Mayr \& Keele, 2000; Moritzetal, 2004; Whitmer \&

67 Banich, 2007). Within this paradigm, participants switch between three tasks, and each currently-

68 relevant task is signaled by a task cue. When switching between tasks A, B, and C, an "ABA"

69 sequence means switching back to a task that was recently performed, and a "CBA" sequence

70 means switching back to a task that was not recently performed. These researchers found that the 
71 ABA sequence had slower RTs than the CBA sequence, demonstrating an $n-2$ repetition cost.

72 When participants were required to switch from Task A to Task B, residual activation of the

73 recently-performed Task A impaired the successful implementation of Task B (Gade \& Koch, 74 2005). In order to overcome this impairment, Task A must be inhibited (Mayr \& Keele, 2000).

75 When participants are required to return to Task A, this task remains inhibited, and its execution 76 takes longer (Gade \& Koch, 2008). Thus, task-related stimulus dimensions are targeted by 77 inhibitory processes once these dimensions are actively abandoned (Koch et al., 2010).

78 Furthermore, $\mathrm{n}-2$ repetition costs have been seen in classification tasks, response modes, 79 language-defined response sets, and cue-target relations. These studies suggest that $\mathrm{n}-2$ repetition costs are markers for inhibitory processes across various levels within a task set (Koch 81 et al., 2010).

82 Previous studies have investigated the role of S-R mapping in generating backward inhibition, 83 and they found that backward inhibition depends on the task switch's response selection stage by 84 adapting the paradigm to include a go/no-go manipulation (Schuch \& Koch, 2003). Additionally, 85 overlap in a response set affects task inhibition (Gade \& Koch, 2007), suggesting that when 86 competing tasks share response sets (e.g., the same left and right key presses), the competition 87 between tasks increases and triggers task inhibition to resolve this competition. They found 88 overlap in the response set, thereby influenced the occurrence of task inhibition, reflecting a n-2 89 repetition cost. However, in a task-switching study, Houghton, Pritchard, and Grange (2009) 90 found that complex (spatially incongruent) S-R mapping reduced overall RTs, but did not affect 91 backward inhibition. Therefore, studies on the effect of S-R mapping on backward inhibition and 92 overlap in a response set on task inhibition have delivered inconsistent results. Moreover, S-R 93 mapping is a subprocess of response selection, and the left inferior frontal gyrus was found to be 
94 involved in controlled response selection (Goghari\& MacDonald,2009). Backward inhibition

95 also involves response selection (Schuch \& Koch, 2003), and it has been suggested that

96 backward inhibition processes are mediated via networks consisting of extra striate occipital

97 areas, the temporo-parietal junction, and the inferior frontal gyrus (Zhang et al., 2016). The

98 overlap of S-R mapping and backward inhibition in the inferior frontal gyrus suggests that they

99 may affect each other while competing for the same cognitive resources. However, few studies

100 have focused on the impact of S-R mapping on backward inhibition, or have fully explained the

101 influence of S-R complexity on task-set inhibition in one experimental setting. Therefore, we

102 manipulated and generated different S-R complexities in order to investigate the implication of

103 task-set inhibition on task switching. S-R complexity refers to the different stimulation and

104 response difficulties. Whether the response mapping was cued or not reflected the response

105 difficulty, and whether the task was univalent or bivalent reflected the stimulation difficulty.

106 Some studies have also tested this manipulation to define S-R complexity (Iveson, Tanida, \&

107 Saito, 2016). Therefore, we manipulated the response mapping and the task values to define four

108 different types of S-R complexities: univalent stimulus with cued response mapping, univalent

109 stimulus with uncued response mapping, bivalent stimulus with cued response mapping, and

110 bivalent stimulus with uncued response mapping. However, it was too difficult for participants to

111 complete the bivalent stimulus with uncued response mapping, which created a floor effect in

112 our pre-experiment. Therefore, we used the three other types to define S-R complexity in a

113 formal experiment. The easy condition had a univalent task value, only one dimension needing to

114 be discriminated (e.g., color), and a cued response mapping, creating the simplest S-R

115 complexity. The moderate condition also had a univalent task value and only one dimension

116 needing to be discriminated (e.g., color), but the response mapping was uncued, making the S-R 
117 complexity moderate. The difficult condition had a bivalent task value, two dimensions needing

118 to be discriminated (e.g., color + shape), and the response mapping was cued. This S-R

119 complexity was the most difficult. Participants were instructed to discriminate between the

120 stimuli dimensions and make the corresponding response. In this study, we investigated the

121 influence of S-R complexity on task-set inhibition in task switching. We manipulated both

122 factors in the same experiment using the backward inhibition paradigm and by controlling the

123 task sequence and S-R complexity. To implement Task A in Trial n, we inhibited the residual

124 activation of Task $\mathrm{A}$ in the $\mathrm{n}-2$ trial. Therefore, the inhibition was in the ABA sequence. In this

125 study, the $\mathrm{n}-2$ repetition cost illustrated the inhibition effect. When the resources needed for a

126 cognitive activity exceeded an individual's total cognitive resources, this led to cognitive

127 overload and affected the efficiency of the individual's cognitive processing (Chandler \&

128 Sweller, 1991). Because of this, we expected that more difficult S-R complexities would lead to

129 greater switch costs, and that increasing the difficulty of the S-R complexity would weaken the

130 effect of the task-set inhibition.

131 Materials \& Methods

\section{Participants}

133 Thirty-two undergraduate $\operatorname{students}\left(M_{\mathrm{age}}=18.83\right.$ years, $S D_{\mathrm{age}}=0.81$ years $)$ from Shaanxi Normal

134 University were compensated to participate in the experiment. All participants reported normal

135 or corrected visual acuity. They did not know the purpose of the experiment in advance. They

136 signed informed consent that were approved by the Committee on Human Research Protection of

137 the School of Psychology of Shaanxi Normal University (Ethical Application Ref: HR 2019-04-

138 007). 
139 We conducted an a priori power analysis using $\mathrm{G}^{*}$ Power (Faul et al., 2009) in order to determine

140 the sample size before data collection. The sample size was determined by estimating a moderate

141 effect size for our interaction effect $(f=0.2)$ and using a power analysis that set the alpha level at

1420.05 to determine the number of participants needed to achieve a 0.90 power level with a

143 medium-size effect. The power analysis showed that a sample size of approximately 28

144 individuals was sufficient to achieve a power level of 0.90 . Based on this analysis and on

145 previous studies (Grange \& Houghton, 2010; Gade \& Koch, 2014), were cruited 32 participants

146 to participate in our experiment.

\section{Apparatus and stimuli}

148 The stimuli were presented on a 17-in color monitor with a viewing distance of approximately 50

$149 \mathrm{~cm}$. We set the screen resolution at 1,280×1,024 pixels and the refresh rate at $60 \mathrm{~Hz}$. The centric

150 fixation subtended $2.7^{\circ} \times 2.7^{\circ}$ and the target traffic signs subtended $2.7^{\circ} \times 2.7^{\circ}$. The cues varied

151 across the three tasks and were all presented in one outlined square, subtending $2.7^{\circ} \times 2.7^{\circ}$.

152 Before starting the experiment, all participants were orally informed to consider the traffic sign's

153 shape, color, and texture (but not the meaning), and they were also given written instructions. All

154 participants were given the same information and instructions about the experiment. The cues

155 were different for different tasks of varying difficulty. In the easy condition, the cue was a word

156 in Chinese, either 颜色 (color), 形状(shape), or 纹理(texture), and the two corresponding keys

157 on the keyboard were marked below the Chinese word. The cue was presented in the center of

158 the screen to remind the participants to focus on this dimension of the stimulus during the

159 discrimination task. In the moderate condition, the cue was one word in Chinese, either 颜色

160 (color), 形状 (shape), or 纹理 (texture).In the difficult condition, the cue was two words in 
161 Chinese, either 颜色+形状(color+shape), 形状+纹理(shape+texture), or 纹理+颜色

162 (texture+color), and two of the four keys corresponding with the dimensions were marked below

163 the Chinese word ( see Figure 1). The response keys were based on the conjunctions of the two

164 characters. Participants were tasked to discriminate the stimuli dimension and to make the

165 corresponding response. In the easy and moderate conditions, participants needed to discriminate

166 one stimuli dimension (color, shape, texture) and make their response accordingly. For example,

167 during a color task, the " $m$ " key corresponded to the yellow stimuli and the " $\mathrm{z}$ " key

168 corresponded to the green stimuli. In the difficult condition, participants needed to discriminate

169 two stimuli dimensions (color + shape, color +texture, or shape +texture) and make their

170 response accordingly. For example, the " $\mathrm{m}$ " key corresponded to the green triangle, yellow

171 horizontal line, and triangle with a vertical line stimuli. The " $\mathrm{z}$ " key corresponded to the green

172 circle, green vertical line, and circle with a horizontal line stimuli. Two keys were presented on

173 one cue at a time, and all combinations were counterbalanced across participants.

174 Design and procedure

175 The experiment was conducted using E-prime 2.0. We used a3 (task sequence: ABA, CBA, $176 \mathrm{XAA}) \times 3$ (S-R complexity: easy, moderate, difficult) within-subjects factorial design. The labels

$177 \mathrm{~A}, \mathrm{~B}$, and $\mathrm{C}$ were used as placeholders, meaning that each task could be repeated. When the

178 sequence had the same task for the $n$ and $n-2$ trials, the sequence was defined as an "ABA

179 switch sequence" (i.e., ABA, ACA, BAB, BCB, CAC, or CBC). When the sequence had

180 different tasks for the $\mathrm{n}, \mathrm{n}-1$, and $\mathrm{n}-2$ trials, it was defined as a "CBA switch sequence" (i.e.,

181 CBA, BCA, CAB, ACB, BAC, or ABC). Finally, when the sequence had the same task for the $n$

182 and $\mathrm{n}-1$ trials, it was defined as an "XAA repeat sequence" (i.e., ABB, ACC, BAA, BCC, CAA, 183 CBB, AAA, BBB, or CCC). 
184 Each trial started with one fixation presented on a black background for $200 \mathrm{~ms}$. The cue then 185 appeared for $1,000 \mathrm{~ms}$ to remind the participants to focus on this dimension of the stimuli during 186 the discrimination task. Finally, the target was displayed until the participants responded, and an 187 error response was signaled through feedback. Participants were instructed to respond as quickly 188 as possible while maintaining attention and at least $90 \%$ performance accuracy. The next trial 189 started after a blank inter-trial interval of $100 \mathrm{~ms}$. The trials were presented in triplicate, with 190 three trials representing one sequence. The intervals between these triple sequences were 1,500 $191 \mathrm{~ms}$ (one-third of the total sequences) or 3,000 ms (two-thirds of the total sequences) (Dreher \& 192 Berman, 2002). The inter-sequence interval was randomized across the sequences ( see Figure 2). 193 Each participant completed three sessions (easy, moderate, and difficult) for the nine blocks, 194 with 72 sequences per block and three blocks per session. They also completed one 36-sequence 195 practice block per session. Participants were encouraged to take a short break after each block.

196 Given that the difficult task took $50 \mathrm{~min}$ to complete, which was almost the time it took to 197 complete both easy and moderate tasks (60 $\mathrm{min})$. Therefore, in order to avoid fatigue, we divided 198 the easy and moderate tasks into one session (session A) and difficult task into another session 199 (session B). And each participant completed two sessions on two separate days, the order of the 200 easy and moderate tasks in session A and the order of the two sessions were counterbalanced 201 across participants.

\section{Results}

203 The data were analyzed using SPSS18.0. We only analyzed the RTs for correct responses. The 204 first set of three trials every per block as warming up trials was excluded and RTs with more than 205 three standard deviations were excluded from the analyses; approximately $3.8 \%$ of the trials were 206 discarded. All participants performed at levels of $>90 \%$ accuracy in each condition across all 
207 experiments. We used bivariate correlations between the RTs and accuracy data $(r=0.083, p=$ 208 0.652) and found no significant correlations between them, suggesting that the RT results could 209 not be attributed to the tradeoff between response speed and response accuracy.

210 RT data were submitted to a $3 \times 3$ repeated measures analysis of variance (ANOVA), using task 211 sequence (ABA, CBA, and XAA) and S-R complexity (easy, moderate, and difficult) as the 212 factors ( see Figure 3). The results revealed the task sequence's main effect was significant ( $F$ $213(2,62)=27.86, p=0.000$, and $\left.\eta_{p}{ }^{2}=0.47\right)$. Post hoc tests (LSD) showed that RTs in the XAA 214 sequence were significantly faster than those in the ABA sequence $(p<0.001)$ and the CBA 215 sequence $(p<0.001)$, generating a switch cost. The main effect of S-R complexity was also 216 significant $\left(F(2,62)=9.41, p<0.001, \eta_{p}{ }^{2}=0.23\right)$. Post hoc tests showed that RTs in the easy 217 condition were significantly faster than those in the moderate $(p=0.013)$ and difficult $(p=0.003)$ 218 conditions. RTs in the moderate condition were significantly faster than those in the difficult 219 condition $(p=0.007)$, suggesting a valid S-R complexity. More importantly, the interaction 220 between task sequence and S-R complexity was significant $\left(F(4,124)=9.29, p<0.001, \eta_{p}{ }^{2}=\right.$ 0.23). Simple effect analyses showed that in the easy condition, the effect of the task sequence was significant $(F(2,62)=16.90, p<0.001)$. Post hoc tests with LSD showed RTs in the XAA 223 sequence were significantly faster than those in the ABA sequence $(p<0.001)$ and the CBA sequence $(p=0.001)$, yielding a switch cost. Moreover, RTs in the CBA sequence were 225 significantly faster than those in the ABA sequence $(p=0.045)$, reflecting an $\mathrm{n}-2$ repetition cost. In the moderate condition, the effect of the task sequence was significant $(F(2,62)=15.12, p<$ 227 0.001), and post hoc tests showed that RTs in the XAA sequence were significantly faster than those in the ABA sequence $(p<0.001)$ and the CBA sequence $(p<0.001)$, yielding a switch cost. However, the RTs did not significantly differ between the CBA and ABA sequences ( $p=$ 
230 0.632). In the difficult condition, the effect of the task sequence was significant $(F(2,62)=$

231 17.12, $p<0.001$ ), and post hoc tests showed that RTs in the XAA sequence were significantly

232 faster than those in the ABA sequence $(p=0.003)$ and the CBA sequence $(p<0.001)$, yielding a

233 switch cost. Moreover, RTs in the ABA sequence were significantly faster than those in the CBA

234 sequence $(p=0.003)$, reflecting an $\mathrm{n}-2$ repetition facilitation.

235 Error rate data (Table 1) were also submitted to a $3 \times 3$ repeated measures ANOVA, using task 236 sequence (ABA, CBA, XAA) and S-R complexity (easy, moderate, difficult) as the factors. The

237 main effect of the task sequence was significant $\left(F(2,62)=6.15, p=0.004, \eta_{p}{ }^{2}=0.17\right)$. Post

238 hoc tests showed that error rates in the CBA sequence were significantly greater than those in the 239 ABA sequence $(p=0.003)$ and the XAA sequence $(p=0.032)$. The main effect of S-R 240 complexity was also significant $\left(F(2,62)=5.43, p=0.007, \eta_{p}^{2}=0.15\right)$. Post hoc tests showed 241 that error rates in the difficult condition were significantly greater than those in the moderate ( $p$ $242=0.001)$ and easy $(p=0.045)$ conditions. The two-way interaction between task sequence and S$243 \mathrm{R}$ complexity was not significant $\left(F(4,124)=2.41, p=0.053, \eta_{p}{ }^{2}=0.07\right)$. Simple effect

244 analyses showed that in the easy condition, the effect of the task sequence was not significant $(F$ $245(2,62)=0.25, p=0.778)$. In the moderate condition, the effect of task sequence was significant $246(F(2,62)=3.58, p=0.034)$, and post hoc tests showed that error rates in the CBA sequence 247 were greater than those in the $\operatorname{ABA}$ sequence $(p=0.001)$. In the difficult condition, the effect of 248 the task sequence was significant $(F(2,62)=6.32, p=0.003)$, and post hoc tests showed that 249 error rates in the CBA sequence were larger than those in the ABA sequence $(p=0.012)$ and the 250 XAA sequence $(p<0.004)$.

251 To verify the stability of the $\mathrm{n}-2$ repetition facilitation in the difficult condition, we recruited 252 another 32 participants ( $M_{\text {age }}=19.53$ years, $S D_{\text {age }}=0.83$ years $)$ from Shaanxi Normal University 
253 to conduct an additional experiment with the difficult condition. Only RTs for correct responses

254 were analyzed and RTs of more than three standard deviations were excluded from the analyses;

255 thus, approximately $4.1 \%$ of the trials were discarded. RTs and error rate were submitted to a 256 one-way ANOVAs with task sequence (ABA, CBA, XAA) as factor. The ANOVAs conducted 257 on RTs showed a significant main effect of the task sequence $\left(F(2,62)=7.02, p=0.002, \eta_{p}{ }^{2}=\right.$ 258 0.19). Post hoc tests with LSD showed that RTs in the XAA sequence ( $M=936.47 \mathrm{~ms}, S E=$ 259 60.88) were significantly faster than those in the CBA sequence $(M=1012.65 \mathrm{~ms}, S E=75.86)(p$ $260=0.005)$, yielding a switch cost, and the ABA sequence $(M=953.76 \mathrm{~ms}, S E=63.33)$ were 261 significantly faster than those in the CBA sequence $(M=1012.65 \mathrm{~ms}, S E=75.86)(p=0.02)$, 262 reflecting an $\mathrm{n}-2$ repetition facilitation. The ANOVAs conducted on error rate showed that the 263 main effect of the task sequence was not significant $\left(F(2,62)=0.41, p=0.66, \eta_{p}{ }^{2}=0.01\right)$. The 264 results of the additional experiment replicated an $n-2$ repetition facilitation in difficult condition.

\section{Discussion}

266 Our study manipulated S-R complexity and task sequence types in order to investigate the 267 influence of S-R complexity on task-set inhibition in task switching. The RTs were faster in the 268 repeated sequence than in the switch sequence. More importantly, the switch costs differed 269 significantly across the three S-R complexity levels. Specifically, the switch cost was greater in 270 the difficult condition than in the moderate and easy conditions.

271 S-R complexity affected the switch cost. Researchers have suggested that switch costs only occur 272 when the underlying S-R mappings are represented as task sets (Dreisbach, Goschke, \& Haider, 273 2007). In our study, participants were tasked to remember the S-R mappings, strengthen this 274 memory through practice, and then construct the corresponding task set following the 275 instructions. When the cues were presented, participants needed to retrieve the task set, 
276 discriminate the dimensions of the stimulus, and perform a task according to the S-R mappings.

277 In the difficult condition, the S-R complexity was more difficult because the S-R mapping

278 associated two dimensions of the task with a corresponding response key, meaning that the

279 participants needed to correctly select two stimulus characters and one of four response keys.

280 However, S-R mapping was easier in the easy and moderate conditions because it associated one

281 dimension of the task with a corresponding response key, meaning that only one stimulus

282 character and one of two response keys needed to be discriminated. Therefore, both the RTs and

283 switch costs were greater in the difficult condition.

284 In our study, the influence of S-R complexity on task-set inhibition demonstrated different

285 modes via different S-R complexity levels. When two components share common brain regions

286 in the inferior frontal gyrus, they compete for cognitive resources and each component is

287 assigned different resources. The easy condition had a significant $n-2$ repetition cost. According

288 to the account of persisting inhibition, when the $\mathrm{n}-2$ trial (Task A) was completed, Task A

289 remained activated. To overcome the residual activation of Task A, subjects needed to suppress

290 it when required to return to Task A (Trial n). With easier S-R complexity, the participants

291 allocated fewer cognitive resources to discriminate one dimension of the stimulus and select the

292 corresponding keys. Therefore, the brain resources for inhibiting the $n-2$ trial task were

293 sufficient. However, the task was still inhibited and took longer to implement, creating slower

294 RTs for the ABA sequence. In the difficult condition, the $\mathrm{n}-2$ repetition cost was reversed,

295 similar to an $n-2$ repetition benefit, suggesting that residual activation from a previously

296 performed task (i.e., trial $\mathrm{n}-2$, Task A) lessened the accumulating information for the same task

297 in the $\mathrm{n}$ trial. When the $\mathrm{n}-2$ trial task reappeared in the $\mathrm{n}$ trial, participants allocated more

298 cognitive resources to handle the S-R complexity. For example, they would discriminate between 
299 two dimensions of the stimulus and select the corresponding keys. Therefore, the cognitive

300 resources for inhibiting the $\mathrm{n}-2$ trial task were insufficient, causing residual activation to persist.

301 When this $\mathrm{n}$ trial task reappeared, a facilitated effect was obtained. These results were consistent

302 with those of previous studies, suggesting that distracted inhibition is stronger with an increased

303 cognitive load (Conway et al., 1999; Gibbons \& Stahl, 2010).

304 This study had some limitations. First, inhibited ability, a high-level cognitive function, is

305 closely related to the prefrontal lobe, which is not fully developed until adulthood (Luna et al.,

306 2010). Our participants were college students who reached adulthood, but children would not

307 have fully developed inhibited abilities compared to the receded inhibited abilities of older

308 participants. Therefore, future studies should investigate the influence of S-R complexity on

309 task-set inhibition across different age groups from the developmental perspective. Second, the

310 prefrontal cortex, anterior cingulated cortex, pre-supplementary motor area, and posterior

311 parietal cortex were activated during tasks witching (Dove et al.,2000;Duncan, 2001; Johnston et

312 al.,2007;Shi et al.,2010), and the prefrontal lobe was involved in inhibition and S-R mapping.

313 The brain mechanisms of inhibition across different S-R complexity levels require further study.

314

315 Conclusion

316 This study investigated the impact of S-R complexity on task-set inhibition in task switching.

317 The switch costs under difficult conditions were greater than those under moderate and easy

318 conditions, suggesting that S-R complexity affects task switching. Specifically, S-R complexity

319 affected task-set inhibition. The easy condition showed a significant $n-2$ repetition cost, while

320 the difficult condition showed an $\mathrm{n}-2$ repetition benefit, suggesting that an increased S-R

321 complexity does not make the task-set inhibition significant. We suggest that S-R complexity 
322 and inhibition compete for cognitive resources, and that these different resource assignments led 323 to our results.

324

\section{References}

326 Conway ARA, Tuholski SW, Shisler RJ, Engle RW. 1999. The effect of memory load on

327 negative priming: An individual differences investigation. Memory \& Cognition 27(6):1042-1050. DOI: 10.3758/BF03201233.

329

330

331

Dove A, Pollmann S, Schubert T, Wiggins CJ, Yvesvon Cramon D. 2000. Prefrontal cortex activation in task switching: An event-related fMRI study. Cognitive Brain Research 9(1):103-109. DOI: 10.1016/S0926-6410(99)00029-4.

Dreisbach G, Goschke T, Haider H. 2007. The role of task rules and stimulus-response mappings in the task switching paradigm. Psychological Research 71(4):383-392. DOI: 10.1007/s00426-005-0041-3.

Duncan J. 2001. An adaptive coding model of neural function in prefrontal cortex. Neuroscience 336 2:820-829. DOI: $10.1038 / 35097575$.

Gade M, Koch I. 2005. Linking inhibition to activation in the control of task sequences. Psychonomic Bulletin \& Review 12:530-534. DOI: 10.3758/BF03193800. and Cognition 35(4):603-609. DOI: 10.3758/BF03193298. 
341 Gade M, Koch I. 2008. Dissociating cue-related and task-related processes in task inhibition:

342 Evidence from using a 2:1 cue-to-task mapping. Canadian Journal of Experimental 343 Psychology 62(1):51-55. DOI: 10.1037/1196-1961.62.1,51.

344 345

346

347

348

349

350

351

352

353

354

355

356

357

358

359

360

Gibbons H, Stahl J. 2010. Cognitive load reduces visual identity negative priming by disabling the retrieval of task-inappropriate prime information: An ERP study. Brain Research 1330:101-113. DOI: 10.1016/j.brainres.2010.03.022.

Goghari VM, MacDonald AW. 2009. The neural basis of cognitive control: Response selection and inhibition. Brain and Cognition 71(2):72-83. DOI: 10.1016/j.bandc.2009.04.004.

Grange JA, Houghton G. 2010. Heightened conflict in cue-target translation increases backward inhibition in set switching. Journal of Experimental Psychology: Learning Memory and Cognition 36(4):1003-1009. DOI: 10.1037/a0019129.

Houghton G, Pritchard R, Grange JA. 2009. The role of cue-target translation in backward inhibition of attentional set. Journal of Experimental Psychology: Learning Memory and Cognition 35 (2):466-476. DOI: 10.1037/a0014648.

Iveson, MH, Tanida, Y, Saito, S. 2016. Same task rules, different responses: Goal neglect, stimulus-response mappings and response modalities. Psychonomic Bulletin and Review 23(6): 1968-1973.

Johnston K, Levin HM, Koval MJ, Everling S. 2007. Top-down control-signal dynamics in anterior cingulate and prefrontal cortex neurons following task switching. Neuron 53(3):453-462. DOI: 10.1016/j.neuron.2006.12.023.

Peer] reviewing PDF | (2020:07:50584:2:0:NEW 16 Jan 2021) 
361 Kiesel A, Steinhauser M, Wendt M, Falkenstein M, Jost K. 2010. Control and interference in

362

363

364 365

366 367

368

369

370

371

372

373

374

375

376

377

378

379

380

381 task switching - a review. Psychological Bulletin 136(5):849-874. DOI: 10.1037/a0019842.

Koch I, Gade M, Schuch S, Philipp, AM. 2010. The role of inhibition in task switching: A review. Psychonomic Bulletin \& Review 17(1):1-14. DOI: 10.3758/PBR.17.1.1.

Luna B, Padmanabhan A, O'Hearn K. 2010. What has fMRI told us about the development of cognitive control through adolescence? Brain and Cognition 72(1):101-113. DOI: 10.1016/j.bandc.2009.08.005.

Mayr U, Keele JW. 2000. Changing internal constraints on action: the role of backward inhibition. Journal of Experimental Psychology: General 129(1):4-26. DOI: 10.1037/00963445.129.1.4.

Mayr U, Kliegl R. 2000. Task-sets witching and long-term memory retrieval. Journal of Experimental Psychology, Learning Memory, and Cognition 26(5):1124-1140. DOI: 10.1037/0278-7393.26.5.1124.

Meiran, N. 2010. Task switching: Mechanisms underlying rigid vs. flexible self control. In Social Cognition and Social Neuroscience, eds. R. Hassin, K. Ochsner, and Y. Trope (New York: Oxford University Press):217-235.

Meiran N, Chorev Z, Sapir A. 2000. Component processes in task switching. Cognitive Psychology 41(3):211-253. DOI: 10.1006/cogp.2000.0736.

Monsell S. 2003. Task switching. Trends in Cognitive Sciences 7(3):134-140. DOI: 10.1016/S1364-6613(03)00028-7. 
382 Moritz S, Hübner M, Kluwe R. 2004. Task switching and backward inhibition in obsessive-

383

384

385

386

387

388

389

390

391

392

393

394

395

396

397

398

399

400

401

402 compulsive disorder. Journal of Clinical \& Experimental Neuropsychology 26:677-683. DOI: $10.1080 / 13803390409609791$.

Philipp AM, Koch I. 2005. Switching of response modalities. The Quarterly Journal of Experimental Psychology Section A 58(7):1325-1338. DOI: $10.1080 / 02724980443000656$.

Rogers RD, Monsell, S. 1995. Costs of a predictable switch between simple cognitive tasks. Journal of Experimental Psychology: General 124(2):207-231. DOI: 10.1037/00963445.124.2.207.

Schuch S, Koch I. 2003. The role of response selection for inhibition of task sets in task shifting. Journal of Experimental Psychology: Human Perception and Performance 29(1):92-105. DOI: 10.1037/0096-1523.29.1.92.

Schuch S, Koch I. 2004. The costs of changing the representation of action: Response repetition and response-response compatibility in dual tasks. Journal of Experimental Psychology: Human Perception \& Performance 30:566-582. DOI: 10.1037/0096-1523.30.3.566.

Shi Y, Zhou X, Müller HJ, Schubert T. 2010. The neural implementation of task rule activation in the task-cuing paradigm: An event-related fMRI study. Neuro Image 51(3):1253-1264. DOI: 10.1016/j.neuroimage.2010.01.097.

Vandierendonck A, Liefooghe B, Verbruggen F. 2010. Task switching: Interplay of reconfiguration and interference control. Psychological Bulletin 136(4):601-626. DOI: 10.1037/a0019791. 
403 Whitmer A, Banich M. 2007. Inhibition versus switching deficits in different forms of 404 rumination. Psychological Science 18(6):546-553. DOI:10.1111/j.1467$405 \quad 9280.2007 .01936 . x$.

406 Zhang R, Stock AK, Fischer R, Beste C. 2016. The system neurophysiological basis of backward 407 inhibition. Brain Structure and Function 221(9):4575-4587. DOI: 10.1007/s00429-016$408 \quad 1186-0$. 
Figure 1

Figure1. Stimuli used in experiments.

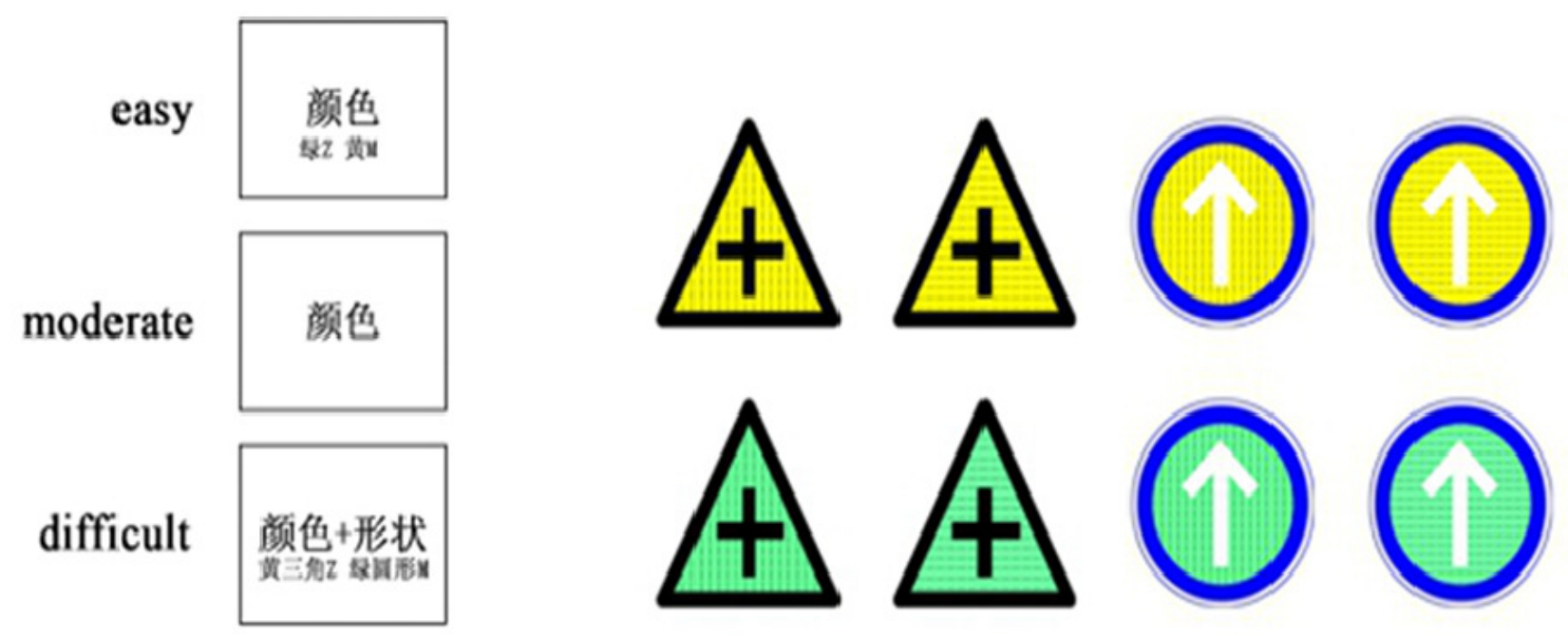




\section{Figure 2}

Figure 2. The time course of a trial in the experiment.




Figure 3

Figure 3. Mean RTs for correct responses in the experiment as a function of task sequence ( $A B A, C B A, X A A$ ) and S-R complexity (easy, moderate, difficult). RT, reaction time. Error bars denote standard errors of the mean. ${ }^{*} p<0.05,{ }^{* *} p<0.01,{ }^{* * *} p<0.001$.

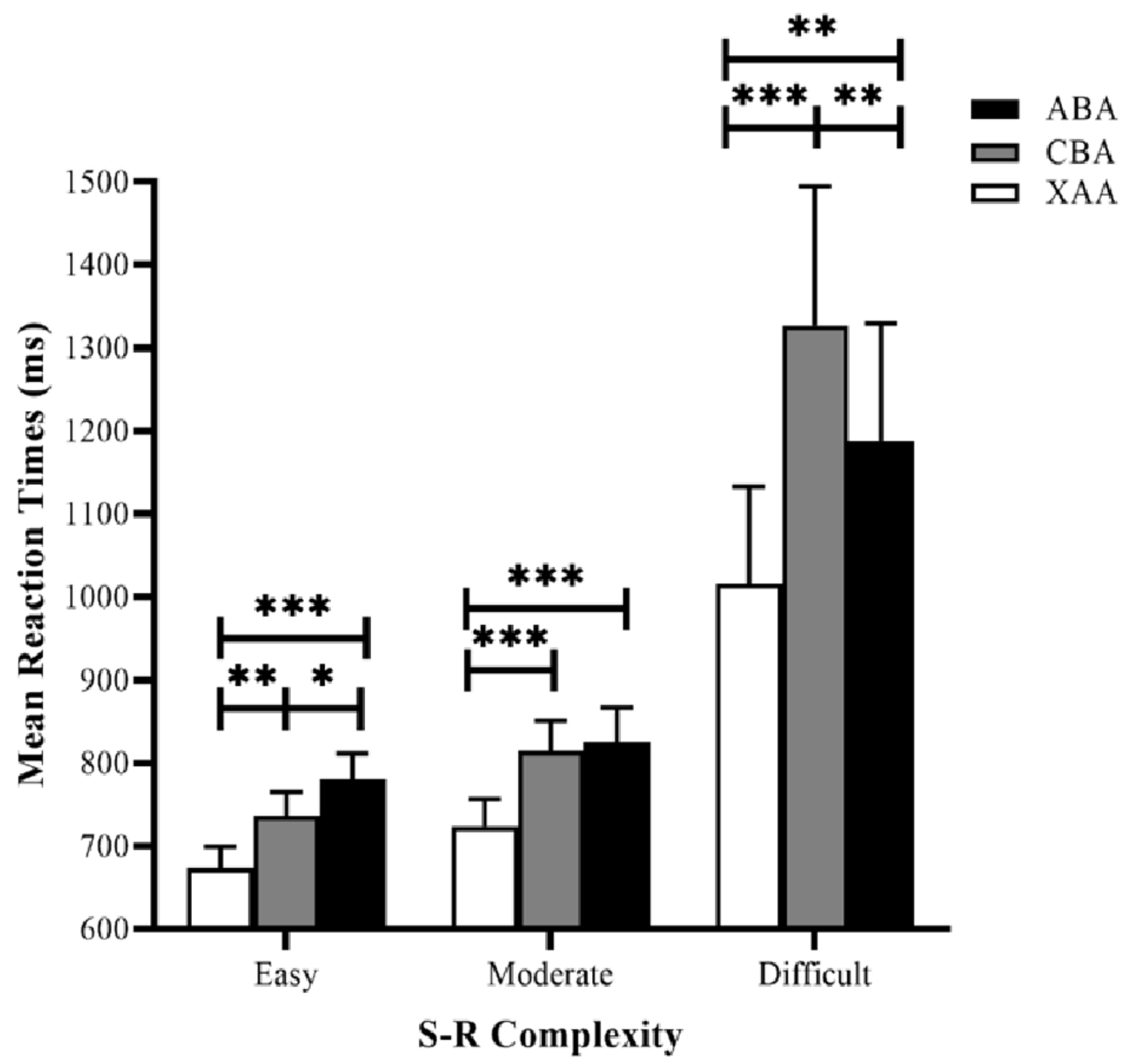




\section{Table $\mathbf{1}$ (on next page)}

able 1. Mean error rates (percentage figures) as a function of task sequence and S-R complexity $(\mathrm{M} \pm \mathrm{SD})$. 
1 Table 1. Mean error rates (percentage figures) as a function of task sequence and S-R

2 complexity (M $\pm S D$ ).

\begin{tabular}{cccc}
\hline Task & \multicolumn{3}{c}{ S-R complexity } \\
\cline { 2 - 4 } sequence & Easy & Moderate & Difficult \\
\hline ABA & $1.07 \pm 1.10$ & $0.78 \pm 0.96$ & $1.35 \pm 1.12$ \\
CBA & $1.07 \pm 1.34$ & $1.20 \pm 1.28$ & $1.95 \pm 1.73$ \\
XAA & $1.17 \pm 1.54$ & $0.94 \pm 0.96$ & $1.39 \pm 1.32$ \\
\hline
\end{tabular}

3

4 